\title{
Laboreal
}

Volume $17 \mathrm{~N}^{\circ} 1$ | 2021

Trabalhar hoje: mudanças, permanências, estratégias, reinvenções

\section{Marzo de 2021: 300.000 muertos en Brasil. La gestión de la pandemia a la luz de las contribuciones de Foucault}

Março de 2021: 300.000 mortes por Covid-19 no Brasil. A gestão da pandemia à

luz das contribuições de Foucault

Mars 2021: 300000 morts au Brésil. La gouvernance de la pandémie à la lumière des apports de Foucault

March 2021: 300,000 dead in Brazil. The governance of the pandemic in accordance with Foucault's contributions

\section{Sandra Caponi}

\section{OpenEdition}

\section{Journals}

Edición electrónica

URL: https://journals.openedition.org/laboreal/17563

DOI: 10.4000/laboreal.17563

ISSN: 1646-5237

Editor

Universidade do Porto

\section{Referencia electrónica}

Sandra Caponi, «Marzo de 2021: 300.000 muertos en Brasil. La gestión de la pandemia a la luz de las contribuciones de Foucault», Laboreal [En línea], Volume $17 \mathrm{~N}^{0} 1$ | 2021, Publicado el 18 junio 2021, consultado el 22 junio 2021. URL: http://journals.openedition.org/laboreal/17563 ; DOI: https://doi.org/ 10.4000/laboreal.17563

Este documento fue generado automáticamente el 22 junio 2021.

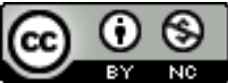

Laboreal está licenciado com uma Licença Creative Commons - Atribuição-NãoComercial 4.0 Internacional. 


\section{Marzo de 2021: 300.000 muertos en Brasil. La gestión de la pandemia a la luz de las contribuciones de Foucault}

Março de 2021: 300.000 mortes por Covid-19 no Brasil. A gestão da pandemia à

luz das contribuições de Foucault

Mars 2021: 300000 morts au Brésil. La gouvernance de la pandémie à la lumière des apports de Foucault

March 2021: 300,000 dead in Brazil. The governance of the pandemic in accordance with Foucault's contributions

Sandra Caponi

1 En el mes de marzo de 2021, un año después de la aparición de la pandemia, Brasil alcanzó el número de 300.000 muertes por Covid-19. En ese marco el presidente Bolsonaro, en una de su muchas lives, habló sobre la inutilidad del distanciamiento social, añadiendo datos de un supuesto estudio alemán referido a las consecuencias indeseadas del uso de máscaras, particularmente por niños. El presidente afirma: “Tengo mi opinión sobre máscaras, que cada uno tenga suya. Pero esperamos un estudio más profundo sobre eso realizado por personas competentes" (Carta Capital, 25/2/2021). El estado de Santa Catarina, donde vivo, alcanzó en el mes de marzo el peor momento de la pandemia, una situación definida por el Secretario de Salud del Estado como siendo de calamidad pública. La ciudad de Florianópolis, capital del estado, se encuentra en riesgo grave, con hospitales colmados, todos ellos con ocupación entre 99\% y 100\%, con falta de suministros y equipos del área de salud, como respiradores y anestésicos, con personas jóvenes muriendo en sus casas por ausencia de camas en Unidades de Terapia Intensiva (UTI), con médicos teniendo que hacer la difícil elección sobre quien será y quien no será atendido, esto es, quien deberá morir y quien podrá vivir. 
2 En ese horizonte de incertidumbre, no podemos dejar de cuestionar qué estrategias biopolíticas (Foucault, 2008) fueron puestas en práctica desde el inicio de la pandemia hasta hoy. Inicialmente observamos con satisfacción como el gobierno del estado de Santa Catarina y el intendente de Florianópolis implantaban medidas de aislamiento, insistían en la importancia de mantener el distanciamiento social y el uso de máscaras. Hablaban de grandes inversiones en salud y de la importancia de higienizar las manos para evitar el contagio. Argumentos que estaban basados en la idea de solidaridad y en la necesidad de cuidarnos entre todos, se insistía en la importancia de permanecer en casa para proteger a aquéllos que estaban obligados a salir a trabajar. Sin embargo, y lamentablemente, ese discurso fue rápidamente abandonado (Caponi, 2020).

Todo parece indicar que existe una aceptación imaginaria de que la pandemia ya fue controlada, que no existe más riesgo de contagio, que es posible circular sin problemas. Se trata de garantizar la libre circulación, aun cuando eso signifique exponer más vidas a riesgo. Una estrategia que parece haber sido muy eficaz para garantizar la reelección del intendente.

4 En todo el mundo, paralelamente al surgimiento de la pandemia, fue posible observar el recrudecimiento de discursos y prácticas racistas, xenófobas y misóginas. Pero, también se multiplicaron las críticas a esas prácticas, ya sea por la divulgación de artículos científicos que dejaron en evidencia las desigualdades de raza y clase de los muertos por Covid (Azria et al., 2020), ya sea por las manifestaciones de movimientos antirracistas, como Black lives Matter Movement, o por la acción de grupos de defensa de los derechos humanos.

5 La actitud crítica, y no el negacionismo, era defendida por Foucault (1995) como siendo la tarea propia del intelectual. Sabemos que para Foucault esa actitud crítica está vinculada a la pregunta kantiana por la Ilustración. Él se pregunta: “¿Qué es ese presente en el cual estamos y desde el cual nos constituimos cómo sujetos?" (Foucault, 1995, p. 7). Dirigir esa actitud crítica a nuestra actualidad significa pensar en el contexto de excepción impuesto por la pandemia de Covid-19. Implica formular la siguiente pregunta: ¿de qué modo están siendo construidas estrategias de gobierno, instituidos o negados discursos y saberes, en tiempos de pandemia?

6 En este artículo propongo analizar diferentes formas de gestión biopolítica en tiempos de Covid-19. Esto es, analizo de qué modo fueron adoptadas diferentes estrategias de gobierno sobre las poblaciones y de qué modo, esas relaciones de poder, se articularon con saberes, discursos y enunciados científicos. Analizo también el impacto de esa forma de administrar la pandemia en el mundo del trabajo.

\section{Biopolítica y gestión de las epidemias}

7 En los primeros días de la pandemia, muchos tuvimos la extraña sensación de estar insertos en una narrativa foucaultiana referida a cuarentenas, aislamiento y pestes. En sus escritos sobre la imposición de cuarentenas para control de la peste en el siglo XVIII, Foucault muestra que el momento de excepción impuesto por una epidemia, exige la adopción de medidas sanitarias concretas, tales como: cerrar las fronteras, reorganizar el espacio, controlar la movilidad, establecer registros, diferenciar enfermos y no enfermos, separar los enfermos y sus familiares, controlar la circulación de objetos y de poblaciones. Sin duda, esos reglamentos no son idénticos a los 
protocolos utilizados en nuestros días. No aparece el mismo espíritu punitivo, ni la vigilancia jerárquica. Sin embargo, e inevitablemente frente a la ausencia de estrategias inmunitarias efectivas, como una rápida y correcta distribución de las vacunas, observamos que muchas estrategias definidas ayer y hoy para evitar el contagio permanecen idénticas. Recordemos que Foucault, refiriéndose al dispositivo disciplinar, como forma de gestión de las epidemias, afirma: "A la peste que es desorden, responde el orden, cuya función es desenmarañar las confusiones provocadas por la enfermedad que se transmite cuando los cuerpos se mezclan. (...) Contra la peste que es confusión la disciplina hace valer su poder de análisis" (Foucault, 1999, p. 201). Ese dispositivo de imposición de normas de aislamiento y cuarentena se opone a la estrategia de expulsión de los leprosos. En este último caso se operaba una división binaria entre unos y otros, lo que configuraba un verdadero abandono, un destierro, una práctica de exilio-clausura, donde los exiliados constituían un grupo indiferenciado de excluidos considerados impuros, sean ellos leprosos, locos, prostitutas. Esa exclusión perseguía el sueño de purificar la ciudad exponiendo esos sujetos a muerte.

8 Cuando la pandemia apareció, muchos levantaron su voz contra el abuso de ese poder disciplinar que pretendía, según entendían, registrar, controlar y punir. Agamben (2020) dijo que era un modo de naturalizar un estado de excepción que ya existía antes de la pandemia, Han (2020) destacó su temor a una disciplina informatizada generalizada, entre otras voces de alarma. Por otro lado, ya en el inicio de la pandemia aparecieron con fuerza los discursos negacionistas de presidentes como Trump y Bolsonaro, los gritos alterados de los defensores de la libertad individual, los que argumentaban contra el uso de máscaras y contra la existencia de la pandemia, que muchos consideran como una simple gripe sin graves consecuencias. Esas voces derribaron rápidamente las estrategias de aislamiento que, tímidamente y lejos de las características autoritarias que caracterizaban a las grandes cuarentenas del siglo XVIII, fueron establecidas para controlar la pandemia. Los opositores a una gestión racional de la pandemia no consiguen entender que estamos, sí, en una situación de excepción. Una situación que exige la creación de estrategias de gobierno destinadas a preservar la vida de las poblaciones, pues, como afirma Foucault, en tiempos de peste "contra un mal extraordinario, el poder se hace visible, inventa engranajes, compartimenta, reticula, inmoviliza" (Foucault, 1999, p. 208).

9 Foucault (2008) retomará la referencia a las epidemias en el marco de los estudios dedicados a la biopolítica. El modelo analizado ya no es el de la exclusión del leproso, ni el dispositivo disciplinar para administrar la peste, sino el modelo de la viruela. Una epidemia cuyo control exige una nueva configuración de las relaciones de poder, una estrategia biopolítica centrada en el dispositivo de seguridad y anticipación de riesgos. En este tercer modelo aparece un nuevo elemento, la variolización y la vacunación, esto es, la conquista de la inmunidad de la población como forma de anticiparse al riesgo de contagio. Foucault insiste en afirmar que esos modelos no se sustituyen, sino que permanecen y se articulan. De modo que, frente a la ausencia de vacunas, las estrategias biopolíticas que deben ser implementadas, sin duda con cambios, alteraciones y adaptaciones, son aquellas viejas estrategias de aislamiento que permiten garantizar el distanciamiento físico para evitar el contagio. Foucault entiende por biopolítica "el conjunto de mecanismos por medio de los cuales aquello que, en la especie humana, constituye sus características biológicas fundamentales puede formar parte de una política, de una estrategia general de poder" (Foucault, 2008, p. 16). 

nacimiento, enfermedad, epidemias, reproducción y muerte, implican determinada "política de la verdad", esto es, determinado modo de articular las relaciones de poder y los discursos, sean ellos verdaderos o falsos. Discursos que, en un determinado momento histórico, aparecen enunciados por los sabes instituidos, por la población en general o por sus autoridades.

11 En el modelo de control de las epidemias, los cálculos de riesgo y las estrategias de anticipación, ocupan un lugar central en la construcción de la biopolítica. De ese modo, es posible anticipar un peligro futuro (real o imaginado) sobre la vida y la salud, dotando de legitimidad y aceptabilidad a las intervenciones sobre las poblaciones, vistas desde una perspectiva biológica. Esos cálculos permiten identificar las regiones y los locales que presentan mayor peligro de contagio, calcular la cantidad de personas infectadas, el nivel de inmunidad existente y, de ese modo, determinar que regiones o ciudades deben ingresar en un esquema de aislamiento mayor e indicar cuándo es posible flexibilizar las medidas. Los cálculos de riesgo constituyen, en fin, un elemento indispensable para organizar las políticas de salud en tiempos de epidemias (Foucault, 2008). la biopolítica aparecen de modo recurrente en diversos textos. Esa recurrencia parece indicar que la salud pública puede ser pensada como una estrategia biopolítica fundamental para que los Estados modernos puedan garantizar el gobierno y la gestión de las poblaciones. Ella permite, al mismo tiempo, administrar los hechos biológicos de una multiplicidad humana, la población, y administrar la salud de cada individuo. Esa biopolítica exige la construcción de un campo de saber referido a las poblaciones compuesto por: las estadísticas poblacionales; los estudios realizados, inicialmente por los higienistas, y luego por sanitaristas; los sabes médicos y biológicos; las ciencias sociales y la epidemiología. La biopolítica deberá hacer uso de instrumentos cuantitativos y de estudios demográficos a partir de los cuales serán definidas las tasas de mortalidad y natalidad existentes y las deseadas, el número de crímenes, de suicidios, de alcoholismo y de locura que cada sociedad debe administrar. En el caso específico de una pandemia, será necesario construir curvas de casos y muertes que permitan realizar comparaciones entre diversas regiones de un mismo país y entre los diferentes países afectados. También, deben ser considerados datos y estadísticas que permitan mostrar que no todas las personas se enferman y mueren del mismo modo. Pues, como ocurre con otras epidemias, existen tasas diferenciales de mortalidad entre ricos y pobres, entre blancos, negros e indígenas, que necesitan ser conocidas y consideradas para que se pueda minimizar esas asimetrías (Ramos, 2020).

13 A partir de esos sabes y discursos, de esos cálculos de riesgo y curvas de normalidad, a partir de la identificación de casos y de la definición de las circunstancias que implican mayor peligro de contagio, fueron legitimándose diversas estrategias biopolíticas de intervención sobre la salud de las poblaciones. Lo que hace aceptables esas intervenciones, muchas veces impositivas, podría ser definido como algo próximo a una promesa de recompensa. Esas prácticas científicamente legitimadas por los sabes reconocidos en cada momento histórico como válidos, independientemente de ser o no estrategias invasivas, se presentan como persiguiendo el único objetivo de maximizar y mejorar la salud de las poblaciones. 


\section{Uma biopolítica exitosa}

14 Si nos detenemos en la situación específica creada en el mundo por la pandemia de Covid-19, podemos observar la aparición de diversos obstáculos que interfieren e interactúan en la construcción de formas efectivas de gestión biopolítica de control de la enfermedad. Existen dificultades económicas, políticas y sociales, vinculadas a la producción de conocimiento y a la producción de vacunas, que impidieron que se establezca un modelo integrado de gestión de la pandemia en todo el mundo. En este sentido, aun existiendo directrices unificadas de la Organización Mundial de la Salud (OMS), cada país definió sus estrategias de control de la pandemia de acuerdo con las posibilidades económicas y con las luchas de poder existentes en cada caso. Es verdad que son pocos los países que pueden considerarse exitosos en el esfuerzo de control de la pandemia, pero hay pocas dudas en reconocer que el modelo biopolítico más adecuado fue el elegido por el gobierno de Nueva Zelandia (Lowy Institute, 2021).

Nueva Zelandia es un país que tiene algunas ventajas en relación a otros, por su condición geográfica y su población. Por ser una isla, el control de entrada y salida de fronteras es más simple, facilitando el aislamiento. Por otra parte, la densidad poblacional del país es baja, con poco más de 5 millones de habitantes, lo que facilita el control y seguimiento de casos. El día 28 de febrero de 2021, cuando Brasil superaba el número de 250.000 muertos, Nueva Zelandia contaba con 65 casos activos, y con un total, desde el inicio de la pandemia, de 2.376 casos de Covid-19. Las muertes reportadas, desde la primera, ocurrida en el día 21 de marzo, hasta el día 28 de febrero, llegaban al número de 26 óbitos (New Zealand Government, 2021b) por Covid-19.

La gestión de la pandemia en Nueva Zelandia reitera muchos elementos de las viejas estrategias biopolíticas narradas por Foucault, articuladas con los nuevos conocimientos científicos hoy existentes. El día 19 de marzo de 2020 el país cerró las fronteras y creó un sistema de 4 niveles de alerta para controlar el riesgo de contagio, con muchas de las características identificadas por Foucault en el dispositivo de seguridad que caracteriza a biopolítica contemporánea (New Zealand Government, 2021a).

17 Ese sistema de alerta fue introducido con el objetivo de gerenciar y minimizar el riesgo de contagio por Covid-19, como un modo de instruir a la población sobre el nivel de gravedad de la pandemia en el territorio y de divulgar las restricciones que deben ser seguidas en cada caso. El sistema de alerta notifica que las medidas pueden ser actualizadas con base en dos criterios: (1) nuevos conocimientos científicos sobre Covid-19; (2) nuevas informaciones sobre la eficacia de las medidas de intervención en Nueva Zelandia y en otros lugares del mundo. Esos niveles de alerta pueden ser aplicados en una ciudad, municipio, territorio. Pueden ser aplicados en nivel regional o nacional, de acuerdo con las necesidades y de acuerdo con el nivel de riesgo. Existen medidas generales de salud pública que se refieren a todos los aspectos: higiene, como lavado de manos y desinfección de superficies; realización de testes; rastreo de casos y autoaislamiento de las personas con síntomas.

18 En los últimos días del mes de febrero de 2021, con 26 muertos y 65 casos activos, Nueva Zelandia pasó del nivel más bajo de riesgo, correspondiente al Alerta 1, a un nivel de riesgo mayor, correspondiente al Alerta 2, después de haber tenido un pequeño aumento del número de casos (de 50 en noviembre de 2020 para 65 en febrero) y un pequeño aumento en el número de muertos (de 25 para 26). Eso significaba que era 
necesario reforzar las estrategias de atención y cuidado para evitar que continuaran aumentando los casos. Las medidas impuestas son muy simples: uso de máscara, distanciamiento de dos metros entre las personas, mantener una buena higiene de manos y desinfección de productos, realización de testes y aislamiento de 14 días por personas que ingresan al país. Se considera que en el nivel de Alerta 2 existe riesgo de que los casos de Covid-19 aumenten y, por ese motivo, se incentiva a continuar registrando el lugar donde la persona estuvo y con quienes se encontró, para ayudar en el rastreo de contactos en caso de necesidad. Puede ser utilizado un aplicativo llamado NZ Covid Tracer en locales públicos, espacios de trabajo y tiendas que permitan hacer ese registro. Por fin, en el nivel de Alerta 1, existe un completo sistema de auxilio económico para individuos, comercios y empresas, que incluye un auxilio a extranjeros residentes en el país.

Hasta alcanzar el nivel de Alerta 1, Nueva Zelandia debió atravesar los niveles anteriores, más graves, cuando la pandemia presentaba mayor riesgo de contagio.

En el día 25 de marzo de 2020, cuatro días después de ser notificada la primera muerte por Covid-19, Nueva Zelandia ingresa en el nivel de Alerta 4, que fue mantenido hasta el día 27 de abril. Ese nivel recibe la denominación de estado nacional de emergencia sanitaria. En ese momento, existía la posibilidad de que la enfermedad no sea contenida, se evalúa que una transmisión continuada e intensa está ocurriendo en la comunidad, con surtos generalizados. Las medidas impuestas en ese período de excepción son muy semejantes a aquellas descriptas por Foucault: las personas son instruidas a quedarse en casa "en su burbuja"; los viajes son radicalmente restringidos; todas las reuniones son canceladas y todos los locales públicos cerrados; los comercios deben ser cerrados, excepto para servicios esenciales; los centros educacionales también deben permanecer cerrados; hay racionamiento de abastecimientos; imposición de aislamiento y cuarentena; priorizando, al mismo tiempo, el fortalecimiento de los servicios de salud. A esas medidas biopolíticas clásicas, donde se articula el dispositivo disciplinar propio de la gestión de las pestes del siglo XVIII, con el dispositivo de seguridad y anticipación de riesgos, debemos añadir tres nuevas estrategias. Ellas son: garantizar auxilio financiero, para que las personas puedan permanecer en sus "burbujas"; las medidas de identificación, con testes aleatorios para la comunidad y con testes en los Centros de salud para las personas con síntomas; y un sistema de rastreo de casos, con uso del aplicativo NZ Covid Tracer. Los códigos de rastreo QR, emitidos por el gobierno de Nueva Zelandia deben ser exhibidos en los locales de trabajo, comercios y en el transporte público para permitir la captación del NZ Covid Tracer. Esas medidas permanecen de manera muy semejante en el nivel de Alerta 3, que se inicia el 27 de abril y permanece hasta el día 13 de mayo.

21 Vemos que, en los meses iniciales de la pandemia, las medidas de gestión utilizadas fueron las más clásicas: cuarentena y aislamiento adecuados a los cálculos de riesgo, pues esas son las únicas medidas posibles ente la inexistencia de una vacuna eficaz. Desde junio hasta hoy las medidas de control fueron adecuándose a los diferentes niveles de riesgo en las diferentes regiones del país, pasando alternativamente del nivel de Alerta 2 al nivel de Alerta 1, y regresado al nivel 2, cuando es necesario. Es posible observar un diálogo permanente y exitoso entre las estrategias de gobierno y las nuevas informaciones y conocimientos científicos, un tipo de gestión que lejos de negar los conocimientos que puedan ser de utilidad para salvar vidas, se compromete con una 
tarea educativa para que la población entienda los riesgos existentes y la necesidad de cuidarse entre todos, ofreciendo soporte financiero cuando necesario.

Ya no se trata de las medidas violentas e impositivas de control de los cuerpos, como el encarcelamiento y el castigo utilizados para controlar las pestes del siglo XVIII. Es posible organizar una gestión biopolítica que maximice la vida y minimice la exposición al contagio y a la muerte con medidas educativas claras, evaluando y divulgando los riesgos, considerando los conocimientos científicos adquiridos, no solo de infectólogos y de sanitaristas, sino también de sociólogos, antropólogos y científicos sociales que contribuyan para minimizar la desigualdad frente a la muerte. La consigna que atraviesa esta biopolítica exitosa es la de la solidaridad colectiva. Parece no existir dudas de que es preciso una gestión colectiva que comprometa a toda la población para contener la pandemia, en este estado de excepción que, de un modo u otro, afecta a todo el mundo. Esa biopolítica se articula en Nueva Zelandia en torno de la idea de que no existe salvación individual, de que la pandemia es un hecho colectivo, y por eso las consignas adoptadas son: "Unidos contra el Covid-19", y "Protéjase usted y proteja a los otros contra el COVID-19" (New Zealand Government, 2021b).

\section{Necropolítica y negacionismo científico}

En las últimas décadas del siglo XX y en las primeras décadas del siglo XXI, las estrategias biopolíticas analizadas por Foucault, preocupadas esencialmente con la estatificación de la vida, eso es, realizadas por estructuras del Estado, empezaron a ser sustituidas por estrategias de gobiernos derivadas de las exigencias del neoliberalismo. Esa nueva configuración política y económica tendrá como resultado fundamental un desplazamiento en el modo de administración y gestión de la vida: sin dejar de ser ejes de intervención y control de los gobiernos, la vida y la salud pasarán a transformarse en asuntos en relación a los cuales cada uno de nosotros es considerado "responsable". Ya sea por los procesos crecientes de privatización de la salud, o como consecuencia de demandas sociales recurrentes, el cuidado con la salud y la anticipación de los riesgos parece haber quedado en nuestras manos. Es justamente esa la consigna utilizada por la municipalidad de Florianópolis en su página de información sobre la situación de la pandemia. Lejos de las consignas ya mencionadas, utilizadas por el gobierno de Nueva Zelandia, "Unidos contra a Covid-19", y "Protéjase usted y proteja a los otros contra el COVID-19", la municipalidad de Florianópolis utiliza la consigna "El control en nuestras manos" (Municipalidad de Florianópolis, 2021), como si una pandemia fuese un problema de gestión individual y no colectiva.

24 Las premisas neoliberales parecen ir en una dirección opuesta a las de la biopolítica implementada en Nueva Zelandia, centrada en la solidaridad y en la protección colectiva. Al contrario, en países como Brasil, el segundo país con mayor número de muertes por Covid-19, lo que predomina en la gestión de la pandemia es una lógica neoliberal de defensa de las libertades individuales sobre todas las cosas: libertad de circular, de vender y comprar, de exponerse y de exponer a los otros al contagio.

Es verdad que esa lógica de defensa de una libertad individual a cualquier precio, contradiciendo las exigencias de un pacto social básico de respeto a la vida, no es un fenómeno exclusivo de este país. Pero, lo cierto es que la biopolítica adoptada por países como Brasil se aproxima a lo que Achille Mbembe identificó como Necropolítica (Mbembe, 2011). Como Foucault afirma en diversos textos, el derecho soberano de 
matar permanece inscripto entre los mecanismos de biopoder de los Estados modernos, una inscripción que ocurre, fundamentalmente, por lo que se denomina racismo de Estado. La obsesión mítica por proteger una supuesta pureza de la raza, encontró en el nazismo el ejemplo, perverso y extremo, de un Estado ejerciendo el derecho de matar. El nazismo se volvió el arquetipo, afirma Achille Mbembe, "de una formación de poder que combinaba el Estado racista, el Estado asesino y el Estado suicidário" (Mbembe, 2011, p. 19). Mbembe da un paso más en esa dirección, antes enunciada por Foucault, cuando afirma que las premisas del exterminio nazista pueden ser encontradas en el imperialismo colonial. Así, aquello que para Foucault constituye el límite extremo e indeseable del biopoder, lo que denomina tanatopolítica, será considerado por Mbembe ya no como un límite extremo, sino como una práctica frecuente y no excepcional, la práctica de exponer ciertos individuos a la muerte. Considera que la noción de biopoder es insuficiente para explicar las formas contemporáneas de sometimiento de la vida al poder de la muerte y que es necesario hablar de necropolítica para indicar la sistemática exposición de ciertos individuos a muertes evitables.

A diferencia de lo que ocurre en Nueva Zelandia, el gobierno nacional y muchos gobiernos provinciales en Brasil sostienen una política de minimización y negación de los riesgos presentados por la pandemia, desconsiderando la gravedad de la enfermedad, exponiendo a la población a la desinformación, al contagio y a muerte. Cuando hablamos de negacionismo, generalmente pensamos en negacionismo científico sin establecer las articulaciones necesarias que ese discurso tiene con la negación de los derechos humanos. En la medida en la que el negacionismo implica el sistemático ocultamiento de la realidad, interfiere directamente en el derecho a la verdad, que es un derecho humano fundamental. Está asociado también a movimientos que niegan situaciones concretas de desaprecio a los derechos, por ejemplo, cuando se niega la existencia del Holocausto, el racismo estructural, o las muertes provocadas por la dictadura militar.

El nuevo discurso negacionista, que se difunde por las redes sociales, cuestiona el valor del conocimiento científico, de los argumentos racionales, de la experiencia adquirida a lo largo de los años, considerándolos como una amenaza desestabilizadora. Independientemente de que esos argumentos se refieran a cuestiones de salud, derechos humanos o a lo preservación del medio ambiente, la idea que prevalece es que todas las opiniones tienen igual valor. De ese modo, el discurso conservador se opone al mismo tiempo a los enunciados científicos y a los derechos humanos, a los que consideran como verdaderas amenazas a los valores tradicionales que defiende: nacionalismo, misoginia, desprecio a las minorías, exigencia de subordinación de trabajadores y desempleados a la lógica empresarial. Hoy, las noticias falsas y las mentiras que se propagan en el whatsapp y por la boca de nuestro presidente, aumentan el miedo y el odio a los opositores, llevando a la pérdida de una realidad compartida, que llega incluso a negar el número de muertos por Covid.

\section{El trabajo en la pandemia: estrategias necropolíticas}

28 La principal premisa del negacionismo científico en relación al Covid-19 es la falsa oposición entre "defender la vida" o "defender la economía", presentadas como polos antagónicos. Para entender la fuerza de esa aparente oposición entre economía y vida, 
es necesario recordar que la pandemia surge en el contexto del neoliberalismo, agudizando aún más la precariedad laboral y las iniquidades sociales ya existentes. Para entender cómo se naturalizó esa lógica que defiende la preeminencia del mercado, aun cuando eso pueda costar la vida de nuestros seres más queridos, es necesario recordar que el neoliberalismo no solo produce servicios y bienes de consumo. Produce también modos de ser sujeto, y el tipo de sujeto que produce es el "capital humano", el empresario de sí, alguien que está fundamentalmente preocupado por la competición y el lucro. En ese marco debemos situarnos para entender por qué motivo se repite esa oposición entre "vida y economía", como si, de hecho, fuese posible imaginar conquistas económicas edificadas sobre cadáveres. La pandemia nos sitúa frente a la debilidad de esa razón neoliberal centrada en la meritocracia, en el éxito y en la construcción del "capital humano".

Para alertar sobre esa falacia, podemos revisar los aislamientos voluntarios de aquéllos que dirigían empresas donde los trabajadores que fueron infectados y murieron fueron sustituidos para continuar produciendo sin que, por supuesto, esos beneficios alcancen a todos igualmente (Vallejo, 2020).

Los defensores del neoliberalismo creen que se beneficiarán con menos Estado, con menos impuestos, con menos dinero público, con menos inversión en educación y salud pública, ahora transformados en espacios de disputa de mercado. Cabe a cada uno de nosotros administrar y anticipar los riesgos, pagar por un plan de salud, por una jubilación, tener un Capital-salud de reserva (Bihr, 2020). Cuando esa lógica neoliberal se enfrenta con un fenómeno dramático como la pandemia de Covid, quedan en evidencia las fragilidades del modelo de salud regulado por el mercado. Aun en un contexto de precarización del Sistema Único de Salud (SUS) y de aumento de contagios y muertes por Covid-19, Bolsonaro y sus seguidores continúan propagando informaciones falsas sobre los efectos mágicos de la cloroquina para prevención y terapéutica, una droga que ya fue excluida de todos los protocolos médicos del mundo. Esa insistencia tiene una funcionalidad ideológica y política muy clara. Pues, existiendo una droga eficaz para prevenir la enfermedad, no habría ninguna justificativa para no retomar a las actividades o para negarse a volver a trabajar.

31 Comencé este texto refiriéndome a la situación calamitosa que viven el estado de Santa Catarina y la ciudad de Florianópolis, con una curva ascendiente de contagios y muertes, y con una situación caracterizada por el secretario de salud del Estado como de calamidad pública, sin que eso haya llevado a las autoridades a modificar significativamente las estrategias de gestión de la pandemia. Ese es el resultado de un estado y de una ciudad que se obstina en negar, siguiendo las enseñanzas del presidente, la existencia de un problema dramático de salud pública, que se obstina en desconsiderar la importancia de las pocas medidas que están a nuestro alcance para contener a pandemia. La situación de Florianópolis replica lo que ocurre en muchas otras ciudades de Brasil.

En la última semana de febrero de 2021, con una situación de caos sanitario, con personas muriendo en sus domicilios, con UTI colmadas y falta de insumos en los hospitales, el gobernador del estado de Santa Catarina presentó medidas muy tímidas de combate a la pandemia. Las medidas consisten en la restricción de actividades después de la media noche y en la adopción de la necesaria medida de lockdown, pero solo por 48 horas, en el final de semana. Aun cuando todos sepamos que ésa es una medida ineficaz, aun cuando los profesionales de la área de la salud y de la educación, 
científicos y epidemiólogos advierten que esa medida no servirá para contener el caos sanitario, la presión empresarial, de los comerciantes, de los sectores de turismo y transporte, inviabilizó cualquier posibilidad de establecer un lockdown por un tiempo de 15 días o más, como sería necesario -paralelamente a la implementación de un auxilio de emergencia, para que los trabajadores puedan realizar el aislamiento, y paralelamente a la agilización de la compra y distribución de vacunas en el estado. En el mes de marzo las medidas fueron alteradas y las restricciones prácticamente dejaron de existir, después de diversas negociaciones y presiones de los empresarios locales.

Sabemos que las medidas de aislamiento y distanciamiento no pueden ser adoptadas por todos, que el transporte público es una fuente efectiva para el contagio y la propagación del virus. Obviamente, muchas personas no pueden parar de trabajar, ni realizar sus tareas de manera remota. Por ese motivo, es preciso que las medidas de asistencia económica y de protección a las poblaciones vulnerables se mantengan hasta el fin de la pandemia.

\section{Consideraciones finales}

Las estrategias biopolíticas analizadas por Foucault en relación a otras epidemias permiten entender los motivos por los cuales, en el contexto de excepción impuesto por la Covid-19, muchos países se vieron obligados a imponer restricciones a sus poblaciones, muchas veces enfrentando situaciones de oposición y resistencia. Esas estrategias destinadas a preservar la vida y disminuir las muertes, están centradas en el control de la circulación, uso de máscaras, aislamiento y lockdown. Esas intervenciones aparecen como inevitables hasta que podamos contar con la generalizada distribución y con la aplicación eficaz de vacunas, único modo de garantizar la inmunidad de la población. Algunos países tuvieron más éxito en la implantación de esas biopolíticas y otros menos. Analizamos aquí el ejemplo de Nueva Zelandia, por la excelente forma de administrar la pandemia que fue adoptada en ese país. El Estudio publicado por el Lowy Institut (Lowy Institute, 2021) de Sidney, destaca el ejemplo de Nueva Zelandia como siendo el modelo más eficaz de control, al mismo tiempo que considera la gestión de la pandemia en Brasil como siendo la peor del mundo.

En el mes de marzo de 2021 Brasil alcanzó el número de 300.000 muertos por Covid-19. Todos sabíamos que llegaríamos a ese número macabro, así como todos sabemos que ese número continuará aumentando, pues poco está siendo hecho para controlar a pandemia. Muchas de esas muertes podrían haber sido evitadas con acciones concretas que ya eran conocidas por todos. Lo cierto es que esas muertes evitables no ocurrieron por acaso, ocurrieron por las decisiones equivocadas adoptadas por el gobierno federal y por los gobernadores e intendentes aliñados a sus ideas. El mundo entero está espantado con las manifestaciones del presidente contra el distanciamiento social, contra el uso de máscaras y con su lentitud para adquirir las vacunas. Observamos que la gestión de la pandemia realizada por el gobierno Bolsonaro tiene todas las características de aquello que Achille Mbembe definió como necropolítica, una política de exaltación y de exposición sistemática a muerte.

La desigualdad frente a la muerte es una característica de esa necropolítica. En Brasil, mujeres y hombres negros perdieron con mayor frecuencia sus trabajos en la pandemia, tienen una tasa de desocupación mayor, y muchos se encuentran en situación de vulnerabilidad, viviendo en habitaciones precarias y con dificultad para 
adherir al aislamiento. Las muertes por Covid-19 fueron y continuarán siendo mayores en la población negra y entre los trabajadores más pobres, permanentemente expuestos al contagio, obligados a desplazarse en transportes repletos y sin poder contar con un auxilio de emergencia para poder realizar las medidas de aislamiento, como ocurre en otros países.

La inexistencia de una política general que establezca las medidas de control de la pandemia, la defensa de la falsa oposición entre defender la vida o la economía, el discurso negacionista que desacredita sistemáticamente los argumentos científicos, la insensibilidad y falta de empatía de los gobernantes, la ineficacia para comprar las vacunas, entre otras cuestiones, han contribuido para que la gestión de la pandemia en Brasil pueda ser definida como una necropolítica.

\section{BIBLIOGRAFÍA}

Agamben, G. (2020). Contagio. In P. Amadeo (Ed.), Sopa de Wuhan, Pensamiento contemporáneo en tiempos de pandemias (pp.31-34). Buenos Aires: Editorial ASPO.

Azria, E., Sauvegrain, P., Blanc, J., Crenn-Hebert, C., Fresson, J., Gelly, M., ... \& Deneux-Tharaux, C. (2020). Racisme systémique et inégalités de santé, une urgence sanitaire et sociétale révélée par la pandémie COVID-19. Editorial. Gynécologie Obtétrique \& Sénologie, 48(12), 847-849. https:// doi.org/10.1016/j.gofs.2020.09.006

Bihr, A. (2020). França: Pela socialização do Aparato de saúde. In D. Harvey, S. Zizek, A. Badiou, M. Davis, A. Bihr, \& R. Zibechi (Orgs.), Coronavirus e Luta de Classes (pp. 25-30). Piauí/Ceará: Terra sem amos.

Caponi, S. (2020, maio). Covid-19 e quarentena em Santa Catarina: um triste experimento populacional. Blog de História, Ciência e Saúde - Manguinhos. Recuperado de http:// www.revistahcsm.coc.fiocruz.br/covid-19-e-quarentena-em-santa-catarina-um-tristeexperimento-populacional/

Carta Capital (2021, 25 de fevereiro). Bolsonaro usa Live para desinformar sobre o uso de máscaras. Recuperado de https://www.cartacapital.com.br/cartaexpressa/bolsonaro-usa-live-nesta-quintafeira-para-desinformar-sobre-o-uso-de-mascaras

Foucault, M. (1995). Que es la Crítica? Critica y Aufldärung. Daimon. Revista de Filosofia, 1(1), 5-25.

Foucault, M. (1999). Vigiar e Punir (2 $2^{\mathrm{a}}$ edição). Petrópolis: Vozes.

Foucault, M. (2008). Segurança, território e população. São Paulo: Martin Fontes.

Han, B.-C. (2020). La emergencia viral y el mundo de mañana. In P. Amadeo (Ed.), Sopa de Wuhan, Pensamiento contemporáneo en tiempos de pandemias (pp. 97-112). Buenos Aires: Editorial ASPO.

Lowy Institute (2021). COVID Performance Index: Deconstructing pandemia responses. Recuperado de https://interactives.lowyinstitute.org/features/covid-performance/

Mbembe, A. (2011). Necropolitica. São Paulo: N-1. 
New Zealand Government (2021a). New Zealand COVID-19 Alert Levels. Recuperado de https:// covid19.govt.nz/alert-system/

New Zealand Government (2021b). Unite against Covid-19. Covid-19 information. Recuperado de https://covid19.govt.nz/health-and-wellbeing/protect-yourself-and-others/

Prefeitura de Florianópolis (2021). Covidômetro. Recuperado de https://

covidometrofloripa.com.br/

Ramos, R. (2020, 12 de novembro). Maior pesquisa sobre Covid-19 conclui que negros, asiáticos e latinos têm maior risco de contrair vírus do que brancos. Jornal o Globo. Recuperado de https:// oglobo.globo.com/sociedade/coronavirus/maior-pesquisa-sobre-covid-19-conclui-que-negrosasiaticos-latinos-tem-maior-risco-de-contrair-virus

Vallejo, G. (2020). Biopolíticas de la pandemia. Blog Epidemias e Saúde Global. Recuperado de https://sehmepidemiassaludglobal.wordpress.com/2020/11/18/biopoliticas-pandemia-vallejo/

\section{AUTOR}

\section{SANDRA CAPONI}

https://orcid.org/0000-0001-8180-944X

Departamento de Sociologia e Ciência Política da Universidade Federal de Santa Catarina - UFSC, Rua Esteves Jr 605. Ap 1414, Florianópolis SC Brasil. CEP: 88015-130

sandracaponi@gmail.com 